\title{
Análise do Sistema de Gerenciamento dos Resíduos de Serviços de Saúde do Hospital Regional de Itacoatiara-AM
}

\section{Analysis of the Health Services Waste Management System at the Regional Hospital of Itacoatiara-AM}

Igor Gabriel de Oliveira Souza - Mestrando em Gestão e Regulação de Recursos Hídricos - ProfÁgua, pela Universidade do Estado do Amazonas (UEA). E-mail: eng. igorgabrielsouza@gmail.com

Bruno Ferezim Morales - Doutorando em Ciências Biológicas (Zoologia) pela Universidade Estadual Paulista "Júlio de Mesquita Filho" (UNESP). Professor da Universidade Estadual do Amazonas (UEA).E-mail: brunomorales@ufam.edu.br

Rodrigo Couto Alves - Mestre em Ciências do Ambiente e Sustentabilidade na Amazônia. pela Universidade Federal do Amazonas (UFAM). Professor da Universidade Federal do Amazonas (UFAM). E-mail: rcouto@ufam.edu.br

\section{Resumo}

O objetivo do estudo foi avaliar o sistema de gerenciamento de resíduos de serviços de saúde - RSS do Hospital Regional José Mendes, Itacoatiara-AM. Foram realizadas a caracterização do estabelecimento, a composição gravimétrica de RSS e a verificação da eficácia do sistema de gerenciamento através de observação direta in loco, registro das informações e análise de documentos internos. Foram identificados os pontos do sistema que não atendiam a legislação pertinente, a estimativa da quantidade de embalagem para acondicionamento de resíduos, dias de maior geração, a proporção de acordo com sua classificação, a relação com os leitos ocupados e problemas de infraestrutura no abrigo externo. Logo, apesar das dificuldades encontradas, este possui uma baixa taxa de geração de resíduos por leito ocupado quando comparado com a literatura, no entanto, a quantidade de resíduos infectantes está próxima de outros valores encontrados por outros autores, corroborando a tendência da alta periculosidade de RSS.

\section{Palavra-chave}

Gravimetria dos Resíduos Sólidos. Indicadores de resíduos. Gerenciamento Integrado. Amazônia.

\begin{abstract}
The paper aimed to analyze the health care waste management system - HCW of José Mendes Regional Hospital in Itacoatiara-AM. Technical visits were performed to analyze the system and its effectiveness, characterizing the establishment and the HCW gravimetric composition. Were employed the methods of direct observation on site, records of information and document analysis. We identified the points of the system that did not comply with legislation, the estimation packaging for waste, days of greater generation, proportion according to its classification and the relation with waste generation and occupied beds. We identified weaknesses in $\mathrm{HCW}$ management and infrastructure issues in the external shelter. Therefore, despite the difficulties found in the management system, we found a low rate of waste generation per occupied bed when compared to literature, however the amount of infectious waste is close to other values found by other authors, corroborating the tendency of high dangerousness of RSS.
\end{abstract}

\section{Keywords}

Gravimetry of Solid Waste. Waste indicators. Integrated Management. Amazon. 


\section{INTRODUÇÃO}

Um dos grandes problemas da atualidade é o aumento da geração de resíduos sólidos, problemas nos sistemas de gerenciamento e atuação em conformidade com a legislação vigente. A definição de resíduos sólidos leva em consideração a característica do resíduo, abrangendo tanto os resíduos sólidos como os semissólidos resultantes de diversas atividades como: doméstica, hospitalar, comercial, agrícola, de serviços e de varrição. Também estão incluídos nesta definição os lodos provenientes de estação de tratamento de água, bem como qualquer líquido contaminado que resulte em alterações da característica de corpos d 'água ou ainda que exijam uma tecnologia inviável em face de melhor tecnologia disponível (ABNT, 2004).

Segundo Zveibil (2001), existem várias maneiras para classificar os resíduos sólidos, sendo que as mais comuns são quanto a sua natureza, origem e quanto aos riscos de contaminação. A classificação de resíduos sólidos envolve a identificação do processo ou atividade que lhes deu origem, de seus constituintes e características, e a comparação destes constituintes com listagens de resíduos e substâncias cujo impacto à saúde e ao meio ambiente é conhecido (ABNT, 2004).

Dentre os resíduos sólidos existem os chamados resíduos especiais provenientes de serviços de saúde (RSS), sejam de hospitais, clínicas médicas, clínicas veterinárias ou de ensino e pesquisa na área da saúde. Os resíduos de serviços de saúde são de natureza heterogênea e estes resíduos de serviços de saúde são muitas vezes chamados de "lixo hospitalar" (GARCIA; ZANETTIRAMOS, 2004). Estes resíduos possuem um alto grau de risco durante o seu manejo, pois possuem características específicas como materiais contaminados perfurocortantes, químicos ou radioativos.

A problemática dos resíduos de serviços de saúde no Brasil, embora inserida e norteada por políticas ambientais, ainda é negligenciada em muitos aspectos, notadamente durante o manejo inicial. Nessa etapa, a maior porcentagem dos resíduos hospitalares é classificada como pertencente ao grupo A (infectantes), demonstrando uma falha na gestão destes, que refletirá em problemas em todas as demais etapas do gerenciamento. De acordo com Ferreira e Anjos (2001), apesar de parte dos resíduos de serviços de saúde não apresentarem risco potencial (resíduos comuns), isso não pode servir de justificativa para que as instituições de saúde não estabeleçam procedimentos gerenciais que reduzam os riscos associados aos demais resíduos gerados nestes estabelecimentos.

O gerenciamento eficaz dos resíduos de serviço de saúde é estabelecido por técnicas, procedimentos, instruções normativas, leis e bases científicas que 
tem como objetivo minimizar a produção de resíduos e destinar de modo correto segundo as legislações pertinentes (RIZZON; NODARI; REIS, 2015).

Segundo Brasil (2018), existem quatro classes e suas respectivas subdivisões que distinguem a periculosidade dos resíduos perigosos, sendo os resíduos hospitalares pertencentes a classe de risco 3 (alto risco individual e moderado risco para a comunidade), que incluem os agentes biológicos que possuem capacidade de transmissão de doenças e patologias humanas ou animais, potencialmente letais que, entretanto, possuem medida de tratamento ou de prevenção.

As principais atividades geradoras de resíduos de serviços de saúde no município de Itacoatiara são as Unidades Básicas de Saúde (UBS), que tem como principal atividade o atendimento ambulatorial, diagnóstico de pacientes e realização de curativos, bem como o Hospital Regional José Mendes, o único estabelecimento hospitalar que realiza atividades de urgência e ambulatorial, com 98 leitos para internação.

Os RSS representam um dos grandes problemas enfrentados pelos administradores hospitalares devido à diversidade de fontes geradoras de resíduos com características específicas, carência de conhecimento sobre a temática e políticas ambientais, manejo inadequado e falta de capacitação dos funcionários e colaboradores que operam estes resíduos com alto potencial de periculosidade.

Deste modo, o presente estudo busca avaliar o sistema de gerenciamento de resíduos de serviço de saúde do Hospital Regional José Mendes do município de Itacoatiara-AM. Por meio da identificação de etapas, pontos de geração, composição gravimétrica dos resíduos e problemas ou lacunas do sistema de gerenciamento foram propostas as melhorias e adequações necessárias ao sistema de gerenciamento de resíduos da saúde da unidade hospitalar do município de Itacoatiara-AM.

\section{PROCEDIMENTOS METODOLÓGICOS}

\section{1 ÁREA DE ESTUDO}

O estudo foi realizado no município de Itacoatiara-AM que, segundo o IBGE (2010), possui uma população de 86.839 pessoas inseridas em uma área territorial de $8.892,038 \mathrm{~km}^{2}$. A cidade possui 17 estabelecimentos de saúde, sendo 11 estabelecimentos de saúde públicos (de esfera de governança municipal) e 06 estabelecimentos de saúde privados. Dentre os 17 estabelecimentos de saúde inseridos no território do município, o Hospital Regional José Mendes (HRJM), localizado na Rua Acácio Leite no 2593, bairro Iraci (Figura 1), foi selecionado 
como objeto de estudo por ser o único hospital existente na cidade e por atender aos critérios de elegibilidade do estudo.

Figura 1 - Localização do hospital José Mendes no município de Itacoatiara - AM

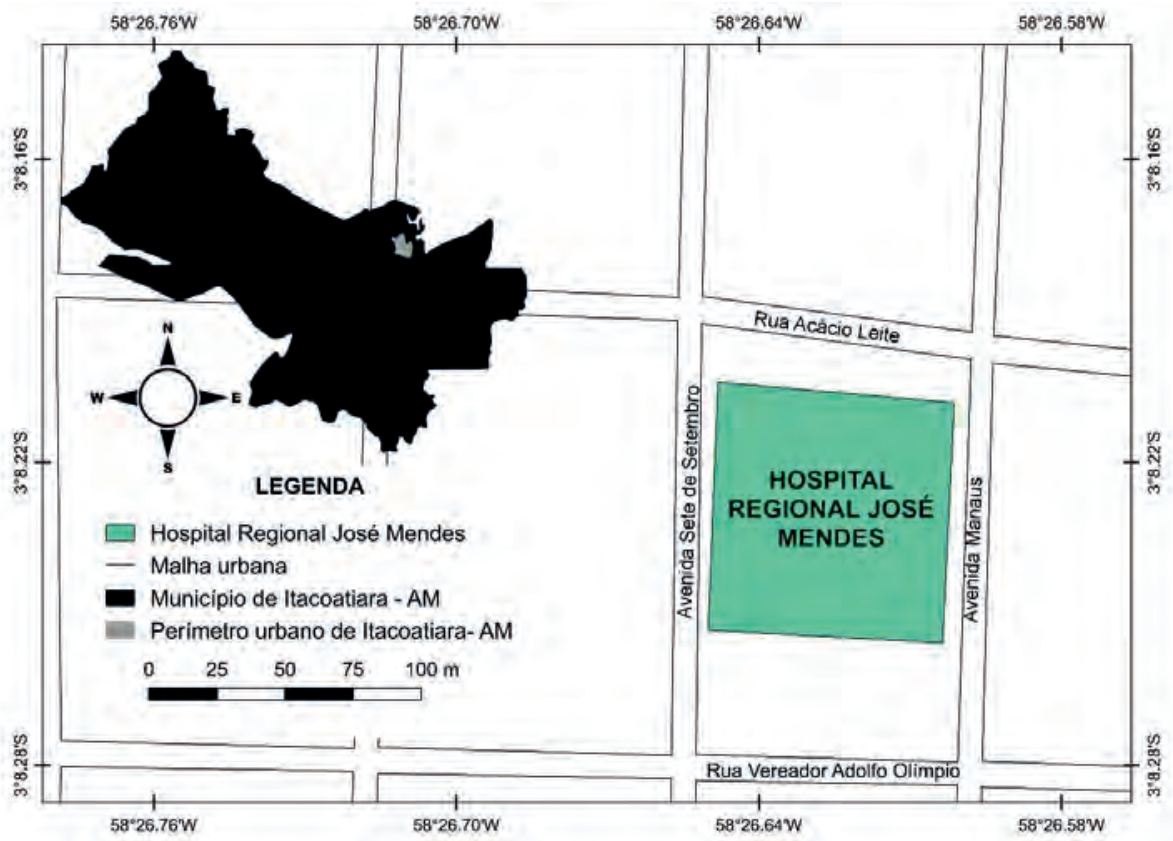

Fonte: os autores (2019).

Os critérios de elegibilidade para a escolha do estabelecimento de saúde Hospital Regional José Mendes foram: abranger uma complexidade maior de serviços de saúde e, consequentemente, maior heterogeneidade de resíduos de serviços de saúde em relação aos demais estabelecimentos de saúde do município; executar atendimentos de urgência e emergência, que resulta na necessidade de atendimento de um conjunto maior e instruções normativas e parâmetros legislações. Além disso, resultados preliminares de um projeto de extensão realizado pela Universidade Federal do Amazonas (UFAM), com o propósito de implantação do Plano de Gerenciamento de Resíduos de Serviços de Saúde, indicaram problemas relativos ao manejo inadequado dos resíduos de serviços de saúde neste ambiente. Segundo os dados do diagnóstico elaborado em 2013, pela gerência do HRJM, a instituição possui um quadro de funcionários com 506 colaboradores, dos quais 254 são da Secretaria de Estado de Saúde do Amazonas (SUSAM) e 252 são da Secretaria Municipal de Saúde (SEMSA).

Por se tratar de um estabelecimento com amplos serviços de apoio, os profissionais e colaboradores do HRJM são de diversas áreas técnicas ligadas 
direta ou indiretamente à área de saúde como, por exemplo, laboratório, banco de sangue, nutrição, lavanderia, higienização, manutenção e setor administrativo. O Hospital Regional José Mendes possui uma média mensal de atendimento de urgência e ambulatorial de 15.000 pessoas, com 21 leitos na clínica médica, 12 leitos na clínica cirúrgica, 26 leitos na maternidade, 8 leitos no centro obstétrico, 24 leitos na clínica pediátrica, 7 leitos no pronto socorro, 08 leitos no centro cirúrgico, totalizando 106 leitos.

\subsection{PROCEDIMENTOS TÉCNICOS PARA COLETAS DE DADOS}

Foi realizada uma pesquisa descritiva e exploratória acerca do sistema de gerenciamento de resíduos sólidos de serviços da saúde do HRJM. A abordagem descritiva caracterizou-se por um estudo detalhado do sistema de gerenciamento dos resíduos, por meio de levantamento de informações e coleta de dados. Para tanto, foram empregados os métodos de observação direta in loco, seguido de registro das informações em diário de campo e coleta de dados, por meio de análises documentais referentes ao gerenciamento de resíduos sólidos, como relatórios de monitoramento, certificado de destinação final, certificado de coleta e manifesto de transporte de resíduos.

A identificação dos pontos de geração de resíduos sólidos de serviços de saúde por unidade funcional do hospital foi realizada por meio de observação dos setores do hospital e procedimentos realizados durante sua operação normal. Foi empregado o registro fotográfico para identificar a forma de segregação, o acondicionamento, o transporte interno nas diferentes unidades, bem como, a estrutura e a operação da central de resíduos, seguido do uso do diário de campo para descrever, detalhadamente, cada etapa do manejo dos resíduos de serviços de saúde, assim como informações qualitativas e quantitativas dos resíduos gerados. Segundo Lira (2014), o diário de campo se faz necessário, em que o pesquisador explora o objeto ou local de estudo constantemente, a fim de produzir e registrar dados fundamentais.

A abordagem exploratória foi baseada no levantamento de dados bibliográficos, referente ao arcabouço legal/normativo pertinente; e dados documentais referentes à destinação final dos resíduos de serviços de saúde que comprovasse o tratamento e destinação ambientalmente adequada, bem como os relatórios de diagnóstico do gerenciamento de resíduos nos últimos anos, com foco na obtenção de dados, descrição de políticas internas, procedimentos e mecanismos que subsidiaram uma descrição minuciosa do manejo dos resíduos de serviço de saúde. 
De forma integrada, as duas abordagens metodológicas empregadas, permitiram a realização de um estudo de caso. Para tanto, foi necessária a inclusão de técnicas e procedimentos em etapas graduais, divididas de acordo com as atividades desenvolvidas em cada unidade (setor) do hospital. Nas unidades do hospital, os resíduos gerados foram classificados, com base na RDC ANVISA n ${ }^{\circ}$ 222/18 e Resolução CONAMA n³58/05, em cinco grupos: A, B, C, D e E. Com base na classificação foi obtida a composição gravimétrica dos resíduos, por meio da pesagem de cada saco plástico armazenado na central de resíduos da unidade hospitalar. A pesagem dos resíduos foi realizada durante 22 dias consecutivos, e os resíduos foram agrupados quanto ao peso total diário por grupo.

Para subsidiar informações para revisão do Plano de Gerenciamento de Resíduos de Serviços de Saúde (PGRSS) (HOSPITAL REGIONAL JOSÉ MENDES, 2017), baseado nas normas técnicas e legislação vigente, foram analisados os procedimentos operacionais e a infraestrutura de apoio das etapas do sistema de gerenciamento de resíduos sólidos.

O diagnóstico resultou da convergência e análise dos dados coletados por todos os instrumentos de pesquisa utilizados, resultando em um diagnóstico das inconsistências frente aos parâmetros sanitários e legais requeridos, que serviram de base para a elaboração de uma proposta com alternativas para adequação do Sistema de Gerenciamento de Resíduos de Serviços de Saúde do hospital.

\section{RESULTADOS E DISCUSSÃO}

A apresentação dos resultados e a discussão do estudo foram divididas em duas partes, sendo a primeira referente a dados resultantes das observações do estabelecimento de saúde e análise de documentos internos; e a segunda parte por meio do levantamento quantitativo dos resíduos originados pelas fontes geradoras no sistema de gerenciamento de resíduos de serviços de saúde do HRJM.

Para melhor compreensão de caracterização do estudo, foi realizado o levantamento quantitativo durante 03 (três) semanas consecutivas, com início no dia 10 de maio e fim no dia 31 de maio de 2017, no qual, foi considerada a classificação de cada resíduo de acordo com a embalagem ou recipiente de armazenamento.

\subsection{CARACTERIZAÇÃO DO GERENCIAMENTO DE RESÍDUOS NO ESTABELECIMENTO DE SAÚDE}

A classificação dos resíduos está diretamente ligada ao manejo adequado, pelo fato de que, cada tipo tem características próprias, sendo desde resíduos 
comuns a potencialmente infectantes. Conhecendo os tipos gerados e os materiais que constituem cada grupo, é possível minimizar o volume de resíduos e, consequentemente, proteger a saúde pública e o meio ambiente.

De acordo com as visitas no local de estudo, os únicos setores que não geram resíduos do grupo A são o almoxarifado e a sala de manutenção. O departamento administrativo consta como gerador deste resíduo, pelo fato de existir uma sala destinada a exames de eletrocardiograma e mapeamento de Holter, de modo que são utilizados materiais como algodão, curativos, luvas de procedimentos, toucas e máscaras. Apesar de ser uma fonte geradora do grupo A, o volume produzido é relativamente baixo, sendo coletado apenas uma vez no dia, se necessário. Os demais setores geram resíduos infectantes, pelo fato de prestarem atendimento aos pacientes ou servir algum tipo de apoio médico.

Os resíduos químicos (grupo B) foram encontrados na clínica médica, laboratório, pronto socorro, maternidade, centro obstétrico, banco de sangue, pediatria, clínica cirúrgica, almoxarifado e administração, sendo que os resíduos químicos como medicamentos vencidos são descartados e armazenados em bombonas no almoxarifado da farmácia. No setor administrativo há o descarte de pilhas na sala de eletrocardiograma e Holter e, durante as visitas, foi verificado que as pilhas são descartadas de forma inadequada, juntamente com os resíduos comuns.

Nota-se que resíduos comuns (grupo D) como, por exemplo, copos descartáveis e guardanapos, foram encontrados em todos os setores da Unidade. Os resíduos perfurocortantes (grupo E) também foram verificados em todos os setores que foram identificados resíduos pertencentes ao grupo A, pelo fato de as atividades desenvolvidas necessitarem dos materiais pertencentes a estes grupos.

Dentre os setores avaliados, foi observada a predominância dos resíduos dos grupos A e D, e constatou-se, a partir das observações, que a cozinha é a maior geradora de resíduos do grupo D, por produzir um grande volume de resto de alimentos, copos descartáveis e embalagens plásticas. O pronto socorro foi enquadrado como maior gerador de resíduos do grupo A, devido ao elevado índice de atendimentos neste setor, em média 500 atendimentos por dia. Tais resultados concordam com o que foi verificado por Nagashima, Barros Júnior e Fontes (2007), que também destacaram o pronto socorro como maior gerador de resíduos infectantes, pela alta concentração de atendimentos e o setor da cozinha como maior gerador de resíduo comum, devido ao funcionamento do refeitório ser lugar de preparo e alimentação dos funcionários, bem como preparação de alimentos para os pacientes.

Não foi possível realizar a quantificação por setores devido à inexistência do quadro de horários para a coleta interna dos RSS, de forma que estes foram 
pesados na central de resíduos do hospital. A falta de treinamento e de capacitação dos funcionários, quanto à forma de classificação dos resíduos, faz com que os resíduos do grupo B sejam descartados juntamente com os resíduos perigosos, tendo tanto a mistura com resíduos infectantes quanto perfurocortantes. Essa dificuldade de quantificar os resíduos do grupo B também foi verificada por Fonseca, Nóbrega e Oliveira (2005) que, da mesma forma, relatam que os resíduos de classe $\mathrm{B}$ (químicos perigosos, resto de medicamentos e outros) foram considerados pertencentes ao grupo A, e associaram tal problema ao precário acesso à informação e a falta de treinamentos específicos dos funcionários.

Desta forma foram apenas quantificados os medicamentos vencidos que encontram-se armazenados em bombonas no almoxarifado de farmácia, representando assim o quantitativo dos resíduos pertencentes ao grupo B. Nesse sentido, observou-se que a média diária de resíduos gerados no hospital é de $49,99 \mathrm{~kg}$, pertencente aos resíduos infectantes (grupo A); 0,28 kg, referente a medicamentos vencidos (grupo B); $87,32 \mathrm{~kg}$, produzido de resíduos comuns (grupo D); e 11, $88 \mathrm{~kg}$, pertencentes aos perfurocortantes (grupo E).

Durante as três semanas em que a composição gravimétrica foi realizada na central de resíduos (Figura 2), constatou-se que não há uma geração de volume contínua no hospital, como observado pela variância das quantidades geradas entre os dias da semana, com marcada alternância entre dias de maior e menor intensidade na produção de resíduo hospitalar.

Figura 2 - Variação da quantidade de resíduos de serviços de saúde do HRJM durante a semana

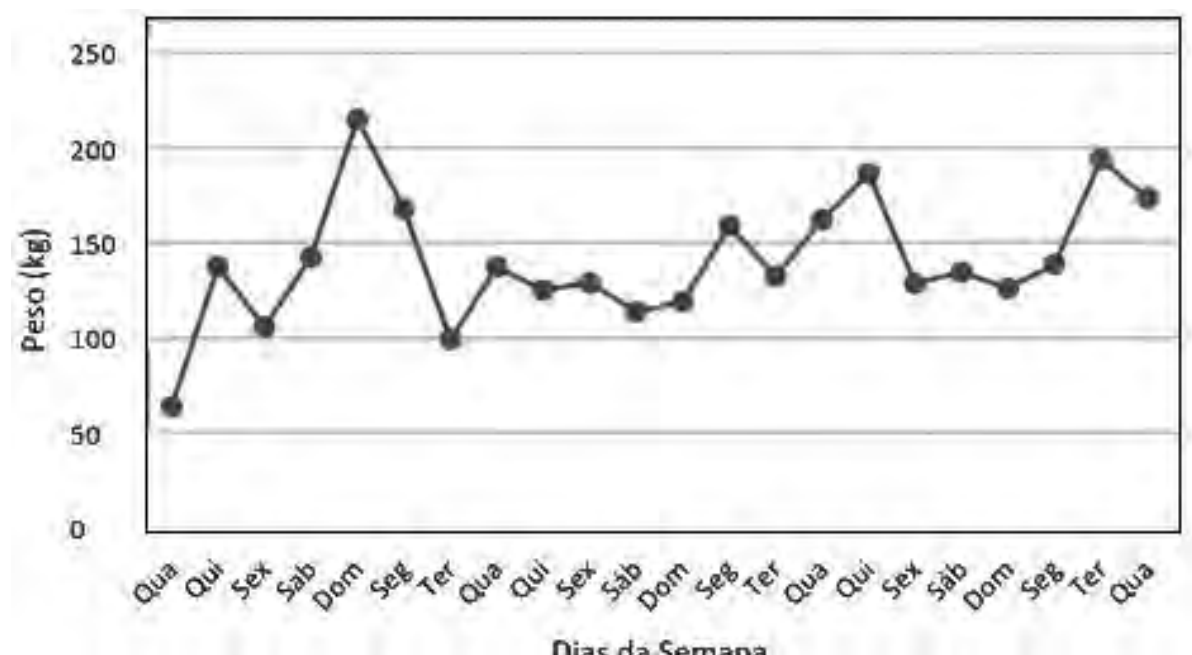

Fonte: os autores (2017). 
Esse quantitativo está interligado à quantidade de atendimentos e internações, sendo maior a produção nos dias de maiores notificações de acidentes. Além disso, observou-se que existe um maior quantitativo de leitos ocupados nesses dias, refletindo assim na geração de resíduos, uma vez que o primeiro dia da semana é considerado como o dia de maior geração de resíduos, devido às internações do dia anterior.

No levantamento quantitativo dos resíduos gerados no HRJM, em novembro de 2016, durante o projeto de extensão, foi verificado que os resíduos produzidos diariamente pertenciam aos grupos A, D e E (Figura 3). Naquele momento, foi verificado que cerca de $45 \%$ dos resíduos gerados no hospital pertenciam à classe dos resíduos perigosos, com obrigatoriedade de tratamento especial, devido à sua periculosidade. Com a elaboração do PGRSS, essa porcentagem foi reduzida para 38\% (29\% destes infectantes). Tais resultados foram condizentes com Ferber (2011), que constatou que 30\% dos resíduos em estabelecimento de saúde foram considerados infectantes e 70\% categorizados como resíduos comuns. Em 2017, com a elaboração do Plano de Gerenciamento de Resíduos Sólidos da Saúde (PGRSS), houve diminuição dos resíduos infectantes (grupo A), aumento dos resíduos comuns (grupo D) e manutenção da proporção dos resíduos perfurocortantes (grupo E).

Figura 3 - Proporção por grupos de resíduos gerados no HRJM durante o monitoramento do estudo (2016) e durante a elaboração do PGRSS (2017)

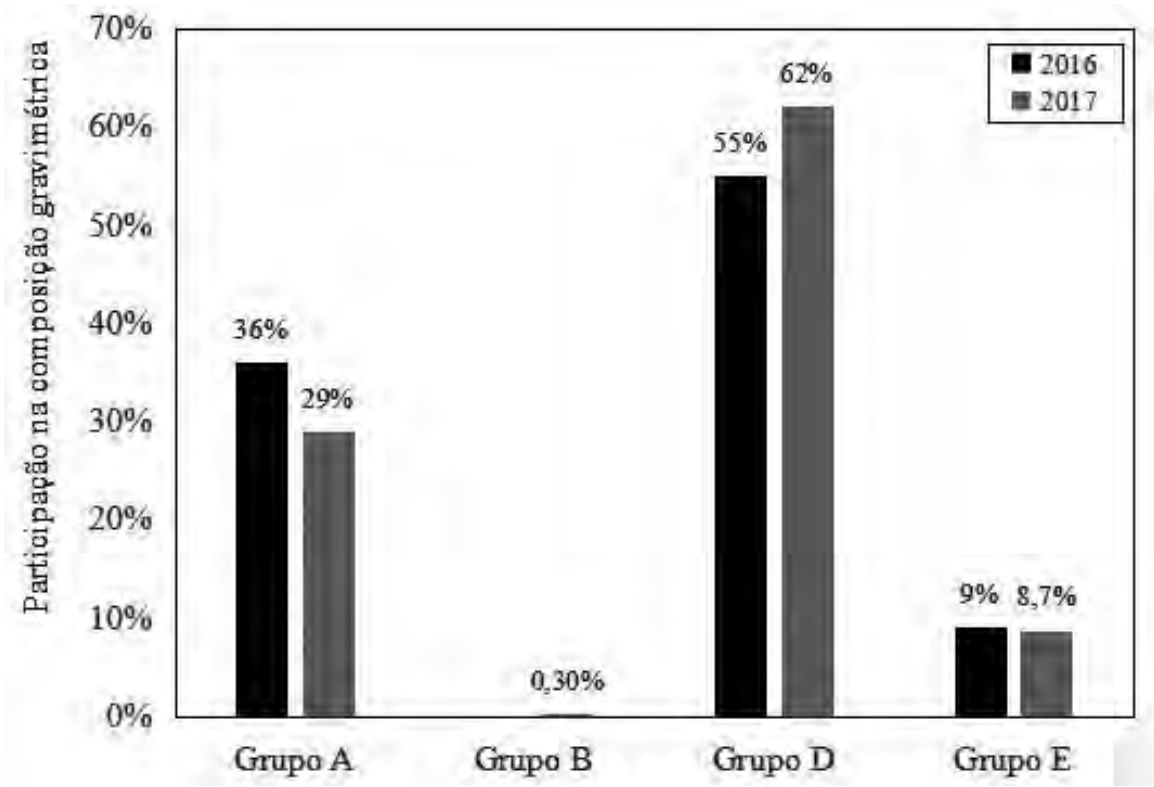

Fonte: os autores (2017). 
Ao comparar os gráficos, observa-se que a redução de resíduos infectantes foi de 7\%, em um período de 4 meses após o término do PGRSS. Essa diminuição pode ser justificada pela adoção de uma segregação mais efetiva, mostrando que uma parte de resíduos comuns era descartada inadequadamente junto com resíduos infectantes. Também se destaca, que essa mudança foi resultante da adoção de fiscalização diária nos setores, por meio da gerência de resíduos de serviços de saúde do HRJM, que passou a adotar orientações aos funcionários ao encontrar inconsistências na segregação.

Com isso, as dúvidas quanto ao descarte de alguns resíduos foram sanadas, aumentando em 7\% a quantidade de resíduos comuns e resíduo perfurocortante, que possui uma característica bem peculiar dos demais resíduos, que continuou estável em sua quantidade. Shiao, McClaws, Huang e Guo (2001) ressaltam que a maioria dos acidentes dentro de estabelecimentos de saúde ocorre entre funcionários da limpeza que manipulam materiais perfurocortantes dispostos inadequadamente pela equipe clínica, responsáveis por cerca de $55 \%$ das injúrias registradas. Os autores também apontam para um dado importante, que mais de $75 \%$ dos acidentes não são reportados e, portanto, há uma lacuna de vigilância neste aspecto. Esse panorama foi essencial para o estabelecimento de ações de treinamento e capacitação dos funcionários do HRJM para a manipulação dos resíduos do grupo E.

Segundo Maders e Cunha (2015), 75\% dos resíduos gerados são correspondentes à classe $\mathrm{D}$ e $25 \%$ são resíduos dos demais grupos (A, B e E), isso quando há uma correta segregação na gravimetria. Isso demonstra que a implantação do PGRSS no HRJM resultará em melhorias contínuas no processo de segregação, que notadamente determina o sucesso das demais etapas de um sistema de gerenciamento de resíduos sólidos

Para a melhor compreensão da geração de resíduos gerados nos setores, foi realizado um inventário, que totalizou a quantidade de resíduos gerados diariamente e a quantidade de leitos ocupados, permitindo verificar a média diária durante os dias da semana e o quantitativo de resíduo que um paciente que ocupa um leito produz de resíduo (Tabela 1).

Para isso, levou-se em consideração o número de atendidos que incide no total de resíduos do dia posterior, uma vez que a pesagem efetuada no período matutino se refere àqueles gerados no início da manhã e no fim do dia anterior. 
Tabela 1 - Geração de resíduos de serviços de saúde por leito ocupado no HRJM

\begin{tabular}{|c|c|c|c|c|}
\hline $\begin{array}{l}\text { DATA / } \\
\text { Ano } 2017\end{array}$ & $\begin{array}{c}\text { Dia da } \\
\text { Semana }\end{array}$ & $\begin{array}{l}\text { RSS Total } \\
\text { (kg/dia) }\end{array}$ & $\begin{array}{l}\text { Total Leitos } \\
\text { Ocupados }\end{array}$ & $\begin{array}{c}\text { Total de RSS Gerado por Leito } \\
\text { (kg res/ leito ocupado dia) }\end{array}$ \\
\hline $10 /$ maio & Qua & 63,72 & 29 & 2,20 \\
\hline $11 /$ maio & Qui & 137,45 & 43 & 3,20 \\
\hline $12 /$ maio & Sex & 105,83 & 46 & 2,30 \\
\hline $13 /$ maio & Sab & 142,41 & 41 & 3,47 \\
\hline $14 /$ maio & Dom & 215,00 & 37 & 5,81 \\
\hline $15 /$ maio & Seg & 167,93 & 50 & 3,36 \\
\hline $16 /$ maio & Ter & 99,00 & 48 & 2,06 \\
\hline $17 /$ maio & Qua & 137,19 & 45 & 3,05 \\
\hline $18 /$ maio & Qui & 125,09 & 53 & 2,36 \\
\hline $19 /$ maio & Sex & 128,92 & 51 & 2,53 \\
\hline $20 /$ maio & Sáb & 113,60 & 49 & 2,32 \\
\hline $21 /$ maio & Dom & 118,90 & 43 & 2,77 \\
\hline $22 /$ maio & Seg & 158,95 & 46 & 3,46 \\
\hline $23 /$ maio & Ter & 132,35 & 49 & 2,70 \\
\hline $24 /$ maio & Qua & 162,16 & 57 & 2,84 \\
\hline $25 /$ maio & Qui & 186,42 & 62 & 3,01 \\
\hline 26/maio & Sex & 128,66 & 56 & 2,30 \\
\hline $27 /$ maio & Sáb & 134,53 & 45 & 2,99 \\
\hline 28/maio & Dom & 126,12 & 53 & 2,38 \\
\hline $29 /$ maio & Seg & 138,61 & 59 & 2,35 \\
\hline 30/maio & Ter & 193,84 & 59 & 3,29 \\
\hline 31/maio & Qua & 173,59 & 52 & 3,34 \\
\hline \multicolumn{2}{|c|}{ Média } & 140,47 & 49 & 2,91 \\
\hline
\end{tabular}

Fonte: os autores (2017).

Os resultados obtidos revelam uma divergência na geração de resíduos durante a semana, bem como o número de leitos ocupados, verificando desta forma que os dias com maior número de leitos ocupados refletiu na geração do resíduo do dia seguinte.

Esta realidade também foi observada por Nagashima, Barros Júnior e Fontes (2007), que constatou que a geração de resíduos não ocorre de forma contínua na semana, sendo observado que o número de internações e atendimentos influenciam, diretamente, na geração de resíduos, mostrando que a taxa de geração de resíduos aumenta de acordo com o número de leitos ocupados. 
Fonseca, Nóbrega e Oliveira (2005) relatam que a taxa de geração de resíduos de serviços de saúde é citada por diversas pesquisas, porém, não se tem uma unanimidade em relação a essa taxa, pois, as características das unidades variam para cada hospital, bem como o tipo de resíduos de serviços de saúde gerados.

Para calcular a quantidade de sacos para acondicionamento de resíduos das classes A, D e E no período de estudo (Tabela 2), utilizou-se a média da quantidade de embalagens para acondicionamento armazenadas diariamente na central de resíduos, tendo uma média de 16 sacos plásticos da cor branca, 31 sacos plásticos da cor preta e 5 caixas para materiais perfurocortantes.

Tabela 2 - Quantidade de embalagem para acondicionamento de resíduos de serviços de saúde do HRJM

\begin{tabular}{l|c|c}
\hline \multicolumn{1}{c|}{ Embalagem } & $\begin{array}{c}\text { Classifi- } \\
\text { cação }\end{array}$ & $\begin{array}{c}\text { Quant. neces- } \\
\text { sária por mês }\end{array}$ \\
\hline $\begin{array}{l}\text { Saco de cor branco leitoso 100 litros com identificação } \\
\text { externa (simbologia infectante - ABNT NBR - 7500) }\end{array}$ & A & 467 \\
\hline Saco plástico de cor preta com capacidade de 100 litros & D & $\mathbf{9 1 8}$ \\
\hline $\begin{array}{l}\text { Embalagem de papelão duplo com plástico (13 litros) } \\
\text { conforme preconizado pela NBR 13853/1997 da ABNT }\end{array}$ & $\mathbf{E}$ & $\mathbf{1 3 8}$ \\
\hline
\end{tabular}

Fonte: os autores (2017).

O levantamento para resíduos do grupo B não foi estimado, por falta de dados reais do quantitativo deste grupo, sendo apenas conhecida a quantidade de medicamentos vencidos.

Esse levantamento de materiais para acondicionamento contribui para o manejo dos resíduos, deixando o hospital ciente de quantos materiais para acondicionamento serão necessários mensalmente, prevenindo a falta dos mesmos e consequentemente acidentes ocupacionais pela ausência das embalagens apropriadas para acondicionamento dos resíduos de serviços de saúde. Além disso, considerou-se um coeficiente de segurança, devido a acidentes como ruptura dos sacos ou mesmo defeito de fábrica. Por meio da quantificação fica evidente a realidade do manejo adotado pelo hospital, pois reflete na proporção de cada resíduo classificado conforme as legislações vigentes.

Rizzon, Nodari e Reis (2015) afirmam que um dos principais dados para a elaboração de um plano de gerenciamento resíduo de serviços de saúde é a quantificação dos resíduos gerados, pois, por meio desse dado é possível dimensionar a quantidade e dimensão de coletores, sacos plásticos, caixas para material perfurocortantes e a central de resíduos. 


\subsection{RECOMENDAÇÕES}

Perante os fatores apresentados, mostra-se essencial a necessidade de otimização de processos internos, procedimentos e infraestrutura voltados ao adequado gerenciamento de resíduos hospitalares na área do estudo, aliado a práticas de gestão ambiental que permitam a redução da quantidade de resíduos a ser tratado. As recomendações são embasadas nas legislações vigentes sobre o gerenciamento de RSS. Estas recomendações visam subsidiar informações para contribuir e adequar as leis pertinentes ao sistema de Gerenciamento de RSS do Hospital Regional José Mendes em seu PGRSS. Para a recomendação do manejo, dividiu-se em etapas que compõem este procedimento e encontra-se em situação precária, sendo apresentado a seguir.

\subsubsection{Segregação e acondicionamento}

Considerando que a segregação dos resíduos, no momento e local de sua geração, permite reduzir o volume de resíduos que necessitam de manejo diferenciado, devem ser realizadas palestras, treinamentos e campanhas educativas sobre a correta separação e acondicionamento dos resíduos gerados, com as suas devidas especificidades para cada setor gerador, pois, os resíduos originados nos setores são devido às atividades desenvolvidas.

Segundo Mustafa, Wenping, Nawaz e Yong (2017), os problemas com a gestão dos RSS afetam vários países em desenvolvimento no mundo e que, se não há recursos financeiros e/ou tecnológicos, a segregação dos resíduos na origem auxilia na diminuição de eventuais impactos ambientais e na redução dos custos de sua destinação.

Deve-se transmitir, de forma clara, as responsabilidades quanto ao corpo técnico de enfermagem, sobre a importância da segregação adequada de cada resíduo e, aos funcionários de serviços gerais, sobre a importância de alocar os recipientes de acordo com o resíduo gerado na unidade, isto é, utilizar saco plástico leitoso e identificado de cor branca para resíduos infectantes (grupo A), recipiente inquebrável e envolvido com saco plástico da cor branca para resíduos químicos (grupo B), saco plástico de cor preta para resíduos comuns (grupo D), bem como usar caixa rígida de dupla camada para perfurocortantes (grupo E).

Os funcionários que atuam no estabelecimento de saúde devem estar cientes do Plano de Gerenciamento de Resíduos de Serviços de Saúde, para que ocorra, dessa forma, uma educação contínua seja implantada e consiga estabelecer o manejo adequado (BRASIL, 2018). 


\subsubsection{Coleta e transporte interno}

Segundo Brasil (2005, 2018), a coleta dos resíduos sólidos de saúde deve ser feita em um carro especialmente projetado para tal serviço, em que este deve ser impermeável, com fácil drenagem, com facilidade de limpeza e com rodas de borracha, para não haver ruídos. Nesse sentido, para adequar-se às resoluções, recomenda-se a compra de dois carros coletores, estilo contêiner, para esta função, sendo um com função de transportar os resíduos comuns (grupo D) e outro para transporte de resíduos perigosos (grupo A e E).

De acordo com a Norma Regulamentadora (NR-32), o transporte dos resíduos e dos recipientes contendo resíduos de serviços de saúde, deve ser realizado de forma que não exista contato do mesmo com outras partes do corpo, além das mãos do trabalhador estarem devidamente protegidas por luvas. Para isso, podem ser feitas adequações dos carrinhos de transporte, eliminação do armazenamento temporário do corredor, com a adoção de uma coleta que recolha os resíduos diretamente da origem e o disponha diretamente na central de resíduos ou rever o local de armazenamento temporário e adaptá-lo às condições exigidas por normas.

A coleta interna deve ter horários definidos, de acordo com cada tipo de resíduos, não podendo ser em horários de grandes fluxos, distribuição de alimentos, roupas limpas e medicamentos. É de suma importância que o funcionário responsável esteja capacitado e devidamente paramentado. Além disso, recomenda-se para a coleta interna:

- Estabelecer turnos, horários e frequência de coleta;

- Sinalizar o itinerário da coleta, de forma apropriada;

- Fazer a manutenção preventiva dos carros para a coleta interna e higienizá-los ao final de cada coleta.

\subsubsection{Armazenamento interno/temporário}

Caso o Hospital Regional José Mendes tenha interesse em fazer da maneira correta, o local de armazenamento não deve estar localizado junto a áreas de armazenamento de alimentos e preparação de refeições e deve estar claramente separado de áreas de armazenamento de material clínico, de consumo de medicamentos e rouparia, no sentido de evitar infecções cruzadas. As leis também deixam claro a não obrigatoriedade de armazenamento interno, caso as fontes geradoras estejam em uma distância relativamente próxima à central de resíduos. 


\subsubsection{Armazenamento externo}

No armazenamento externo, Martins e Mateus (2018) afirmam que a implantação de uma central de resíduos possibilita, além da organização na segregação, a conscientização coletiva na responsabilidade ambiental e ocupacional.

A central de resíduos do hospital possui a divisão para resíduos perigosos (grupo A e E) e para resíduos comuns (grupo D), além de uma sala de materiais de limpeza para a higienização do local. As recomendações estão voltadas à parte física e higiênica como:

- Identificar os compartimentos na parte interna e externa da central de resíduos, utilizando as simbologias determinadas pelas normas;

- Consertar a grelha de proteção da canaleta por onde escoa o efluente da central de resíduos;

- Elaborar um quadro para monitorar os dias e a frequência de limpeza da central de resíduos;

- Colocar maçanetas nas portas danificadas e manter as portas fechadas, para acesso exclusivo aos funcionários da higienização;

- Evitar o lançamento de sacos, para que não cause ruptura;

- Fazer a limpeza na parte externa da central de resíduos após a coleta pela empresa responsável;

- Manter o local trancado, impedindo o acesso de pessoas não autorizadas.

\subsubsection{Tratamento e destinação final}

Recomenda-se ao HRJM entrar em contato com a empresa responsável por fornecer os medicamentos (CEMA), para que seja feita a destinação final dos medicamentos vencidos armazenados no almoxarifado de farmácia, praticando, desta forma, a logística reversa.

Solicitar à empresa responsável pelo tratamento e destinação final as licenças ambiental e sanitária para que se tenha o controle e certeza de que os resíduos estão atendendo às legislações vigentes.

\section{CONCLUSÕES}

Os entendimentos das resoluções vigentes sobre resíduos de serviços de saúde são de suma importância para adequação do manejo de resíduos, de acordo com a realidade local. Seguindo esta linha, todos os objetivos foram 
alcançados, sendo possível caracterizar e identificar as fontes geradoras do hospital, bem como contribuir com informações para aperfeiçoar o sistema de gerenciamento dos resíduos.

As observações e instrumentos utilizados no estudo possibilitaram encontrar os pontos críticos do manejo e fazer recomendações para a melhoria em cada etapa do manejo que se encontrava precária.

Houve dificuldades em quantificar os resíduos pertencentes ao grupo $\mathrm{B}$ devido à única separação destes serem os medicamentos vencidos, que são armazenados no almoxarifado de farmácia, o restante são misturados com resíduos infectantes e perfurocortantes. A quantificação mostrada no estudo é referente aos medicamentos vencidos que ainda não possuem destinação final.

A taxa de geração de resíduos por leito encontrada foi um pouco abaixo da encontrada para países industrializados (3 a $6 \mathrm{~kg}$ resíduo/ leito ocupado), tendo inúmeros variáveis como tamanho do estabelecimento de saúde, quantidades de atendimentos, atividades desenvolvidas que contribuem para tal parâmetro comparativo. A proporção de resíduos infectantes também está próxima dos encontrados por diversos autores, sendo justificado pela incorreta segregação que ocorre no estabelecimento de saúde.

O PGRSS atuou diretamente na melhoria dos processos de destinação final e tratamento dos resíduos, visto que os resíduos do grupo D (com adequações no processo de segregação) eram destinados ao aterro municipal de Itacoatiara onde não geram potencial de periculosidade inata, enquanto os resíduos dos grupos A e E (infectantes e perfurocortantes respectivamente) passaram a ser coletados e transportados por uma empresa de Manaus especializada e certificada para a destinação final e tratamento por incineração destes resíduos especiais.

Neste estudo, também foi possível verificar que o hospital possui uma campanha interna para funcionários e usuários para reduzir a quantidade de copos descartáveis, por meio de incentivá-los à adoção de um copo.

A implantação do PGRSS do hospital é de suma importância para que ocorra a melhoria do sistema de gerenciamento, sendo de imediato o conhecimento deste documento pelos funcionários do HRJM. O sucesso em todas as etapas do manejo de resíduos depende do trabalho coletivo de todos os funcionários, desde o corpo técnico de enfermagem aos funcionários responsáveis pela higienização.

Observou-se a importância de estudos no contexto geral de RSS apontando os benefícios de se adequar ao correto gerenciamento de RSS como a valorização da saúde do trabalhador, a saúde pública e ao meio ambiente. Desta forma o presente estudo subsidiou informações que corroborem para a revisão do PGRSS e contribuam para um sistema de gerenciamento que atende às 
legislações pertinentes. Por fim, espera-se uma melhora no ambiente hospitalar quanto aos RSS e que ocorra uma diminuição dos riscos potenciais à saúde pública, acidentes ocupacionais e impactos ao meio ambiente causadas pelo mau gerenciamento desses resíduos do HRJM.

\section{REFERÊNCIAS}

ABNT. ABNT NBR 10004: resíduos sólidos - classificação. Rio de Janeiro: ABNT, 2004.

BRASIL. Conselho Nacional do Meio Ambiente. Resolução CONAMA n ${ }^{\circ}$ 358, de 29 de abril de 2005. Dispõe sobre o Tratamento e a disposição final dos resíduos de serviços de saúde. Brasília, DF: Conselho Nacional do Meio Ambiente, 2005. Disponível em: http://www.siam.mg.gov.br/sla/download. pdf?idNorma=5046. Acesso em: 12 mar. 2020.

BRASIL. Agência Nacional de Vigilância Sanitária. Resolução RDC no 222, de 28 de março de 2018. Regulamenta as Boas Práticas de Gerenciamento dos Resíduos de Serviços de Saúde e dá outras providências. Brasília, DF: Agência Nacional de Vigilância Sanitária, 2018. Disponível em: https://www.cff.org. $\mathrm{br} /$ userfiles / file/RDC $\% 20$ ANVISA $\% 20 \mathrm{~N} \% \mathrm{C} 2 \% \mathrm{BA} \% 20222 \% 20 \mathrm{DE} \% 20$ 28032018\%20REQUISITOS\%20DE $\% 20$ BOAS $\% 20$ PR $\%$ C3\%81TICAS $\% 20$ DE $\% 20$ GERENCIAMENTO $\% 20$ DOS $\% 20$ RES $\%$ C3\%8DDUOS $\% 20$ DE $\% 20$ SERVI\%C3\%87OS\%20DE\%20SA\%C3\%9ADE.pdf. Acesso em: 12 mar. 2020.

FERBER, M. C. S. Avaliação do sistema de gerenciamento de resíduos sólidos de serviço de saúde do hospital Instituto de Medicina e Cirurgia do Paraná - IMCP. 2011. 89 f. Monografia (Curso de Engenharia Sanitária e Ambiental) - Faculdade de Engenharia Sanitária e Ambiental, Universidade Federal de Santa Catarina, Florianópolis, 2011.

FERREIRA, J. A; ANJOS, L. A. Aspectos de saúde coletiva e ocupacional associados à gestão dos resíduos sólidos municipais. Cad. Saúde Pública, Rio de Janeiro, v. 17, n. 3, p. 689-696, 2001.

FONSECA, E.; NÓBREGA, C. C.; OLIVEIRA, A. G. Produção e taxa de geração de resíduos sólidos de serviços de saúde de hospitais de João PessoaParaíba. In: CONGRESSO BRASILEIRO DE ENGENHARIA SANITÁRIA E AMBIENTAL, 23., 2005, Campo Grande. Anais [...]. Campo Grande: ABES. 2005. p. 1-8. 
GARCIA, L. P; ZANETTI-RAMOS, B. G. Gerenciamento dos resíduos de serviços de saúde: uma questão de biossegurança. Cad. Saúde Pública, Rio de Janeiro, v. 20, n. 3, p. 744-752, 2004.

HOSPITAL REGIONAL JOSÉ MENDES. Plano de Gerenciamento de Resíduos de Serviço de Saúde - PGRSS. Itacoatiara: HRJM, 2017.

IBGE. Censo 2010. IBGE, Rio de Janeiro, 2010. Disponível em: https:// censo2010.ibge.gov.br. Acesso em: 12 fev. 2017

LIRA, B. C. O passo a passo do trabalho científico. Petrópolis: Vozes, 2014.

MADERS, G. R.; CUNHA, H. F. A. Análise da gestão e gerenciamento dos resíduos de serviços de saúde (RSS) do Hospital de Emergência de Macapá. Eng. Sanit. Ambient, Amapá, v. 20, n. 3, p. 379-388, jul./set. 2015.

MARTINS, J. M.; MATEUS, M. P. Proposta técnica para a instalação da central de resíduos de serviços de saúde em projeto modelo de instituição hospitalar. 2018. 98 f. Trabalho de Conclusão de Curso (Graduação em Engenharia Civil) - Departamento de Engenharia Civil, Universidade do Sul de Santa Catarina, Tubarão, 2018.

MUSTAFA, A.; WENPING, W.; NAWAZ, C.; YONG, G. Hospital waste management in developing countries: A mini review. Waste Management \& Research, v. 35, n. 6, p. 581-592, 2017.

NAGASHIMA, L. A.; BARrOS JUNIOR, C.; FONTES, C. E. R. Análise da produção e taxa de geração de resíduos sólidos de serviços de saúde do Hospital Universitário Regional de Maringá. Acta Scientiarum. Technology, [S. l.], v. 29, n. 2, p. 131-139, 2007.

RIZZON, F.; NODARI, C. H.; REIS, Z. C. Desafio no gerenciamento de resíduos em serviços públicos de saúde. Revista de Gestão em Sistemas de Saúde, v. 4, n. 1, p. 40, 2015.

SHIAO, J. S.; MCLAWS, M. L.; HUANG, K. Y.; GUO, Y. L. Sharps injuries among hospital support personnel. J Hosp Infect, v. 49, n. 4, p.262-277, 2001.

ZVEIBIL, V. Z. Manual gerenciamento integrado de resíduos sólidos. Rio de Janeiro: IBAM, 2001. 